\title{
A MEMS-Based Approach that Uses Temperature- Dependent Sensing Responses to Recognize Chemical Targets in Untrained Backgrounds
}

\author{
Baranidharan Raman*, Rupa Shenoy, Douglas C. Meier, Kurt D. Benkstein and Steve Semancik \\ Chemical Science and Technology Laboratory \\ National Institute of Standards and Technology \\ Gaithersburg, MD 20899-8362, USA \\ steves@nist.gov \\ *Current address: Department of Biomedical Engineering, Washington University, St. Louis, MO, USA
}

\begin{abstract}
A major practical challenge for solid state microsensors is the detection of trace chemical species over time and in complex gas-phase backgrounds. We describe a MEMSbased, chemiresistive technology that has succeeded in such problems by combining oxide nanomaterials on microscale platforms, acquisition of dense temperature-dependent response data streams, and novel signal processing methods. Unlike the operation and analysis employed with many electronic noses, our higher dimensional approach captures surface electronic implications of changing adsorptive/reactive phenomena caused by rapid thermal cycling. Here, we demonstrate new capabilities for recognizing toxic targets over extended time periods, even in untrained backgrounds that contain aggressive contaminants at higher concentrations. The approach involves a moving-window, correlation-based methodology to identify chemical events and decouple the foreground conditions from the background.
\end{abstract}

\section{INTRODUCTION}

Temperature is a critical factor in all of chemistry, including the chemical interactions that occur between sensing materials employed in microsensors and the molecules present within an environment being monitored [1-4]. To benefit from the valuable analytical information contained within the temperature-dependent oxide/ambient interactions, we have designed and fabricated, through top-side micromachining, arrays of individually addressable microhotplates [5-7]. The array microelements, which serve as microsubstrates for multiple chemiresisitive sensing oxides, operate with low power consumption and thermal time constants $(\approx \mathrm{ms})$ that are ideal for implementing a variety of temperature programs. Cycling through temperatures rapidly and repetitively generates temporally varying signals that are associated with changing adsorbate populations. Related work [8-10], and our earlier work employing temperature-dependent data streams [11-13], has focused on detecting and recognizing target (volatile organic) chemicals in the presence of known, or pretrained, backgrounds. Here, we present methodology developed to deal with target species detection in untrained background conditions. The target analytes studied were the toxic industrial chemicals (TICs) $\mathrm{NH}_{3}, \mathrm{HCN}$ and $\mathrm{Cl}_{2}$ at immediate-danger-to-life-and-health (IDLH) concentrations. These molecules were presented to the sensor in $20^{\circ} \mathrm{C}$ air with $30 \%$ relative humidity $(\mathrm{RH})$ during the training phase. The testing phase sought to detect these chemicals while relatively high concentrations of interference molecules, such as diesel fuel, Windex [14] cleaner and Clorox [14] bleach, were included in the air-based background.

\section{EXPERIMENTAL AND ANALYSIS METHODS}

\section{A. Device Fabrication and Testing Protocols}

MEMS microhotplate arrays have been used for fabricating chemical microsensor devices [2] and as research tools for investigating temperature-dependent phenomena $[6,15]$. Typically, multiple types of sensing films/materials are used to populate the elements in microsensor arrays in order to gain different information from multiple gas adsorbate-film interactions [12,13]. A 16-element array (see Fig. 1a; a single element is shown at a higher magnification in Fig. 1b) was employed during this study. It was populated with semiconducting films of $\mathrm{SnO}_{2}, \mathrm{TiO}_{2}, \mathrm{SnO}_{2} / \mathrm{TiO}_{2}$, and $\mathrm{TiO}_{2} / \mathrm{RuO}_{\mathrm{x}}$. Four copies of equivalent manufacture for each oxide were all produced by self-lithographic chemical vapor deposition techniques, which have been described elsewhere $[2,12,16]$. For the development work on recognition in untrained backgrounds discussed in this paper, we focused on response signals measured under temperature cycling from a single sensing material $\left(\mathrm{SnO}_{2}\right)$. The morphology typical for these $\mathrm{SnO}_{2}$ films is shown in Fig. 1c. Analytically rich data were acquired by repeatedly cycling the $\mathrm{SnO}_{2}$ devices through a pulsed mode, 32-temperature program with four different base-level temperature sectors (see Fig. 2). These sectors facilitate the development of differing adsorbate populations as a function of time within each program cycle. Conductance 

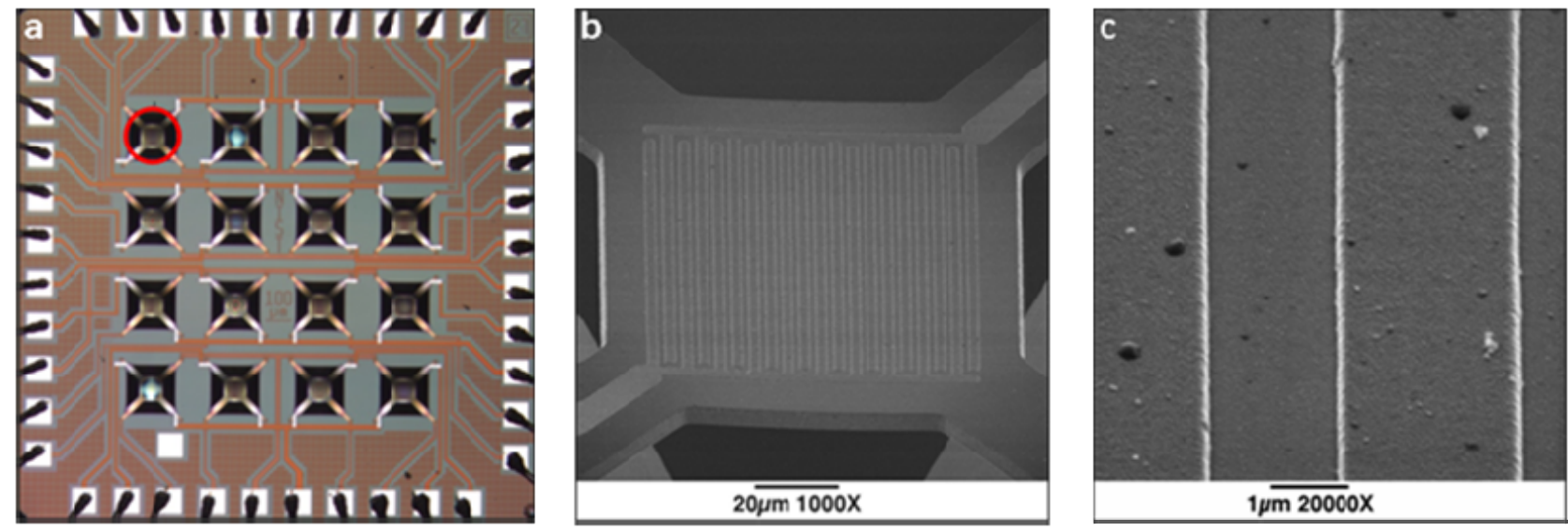

Figure 1. (a) Optical micrograph image of the 16-element array where the red circle denotes a $\mathrm{SnO}_{2}$ sensing element. Scanning electron micrographs of (b) the circled element, with its suspended structure and the electrode combs, and (c) a higher magnification image showing the morphology of the $\mathrm{SnO}_{2}$ sensing film.

measurements made on the $\mathrm{SnO}_{2}$ film elements (at both temperature peaks/ramps and bases) capture the changing surface electronic characteristics to provide information about the composition of the environment being probed.

Metered concentrations of three TICs: $\mathrm{NH}_{3}$ (300 $\mu \mathrm{mol} / \mathrm{mol}), \mathrm{HCN}(50 \mu \mathrm{mol} / \mathrm{mol})$ and $\mathrm{Cl}_{2}(10 \mu \mathrm{mol} / \mathrm{mol})$, were delivered to the microdevice array during the training and testing phases of this study. During the training they were introduced individually in a background of air at $20^{\circ} \mathrm{C}$ with $30 \% \mathrm{RH}$, controlled using zero-grade dry air and a dew-point generator. These target species were delivered to the microsensor array again during the testing phase, which also included periods when interference vapors of diesel fuel, Windex [14] cleaner and Clorox [14] bleach, were introduced individually into the air-based background at concentrations several orders of magnitude higher than the TICs. The total flow in the experiments therefore consists of contributions from an individual target, an interference, humid air and balance dry air. The small-volume test cell in which the sensor array was mounted is positioned downstream of the gas

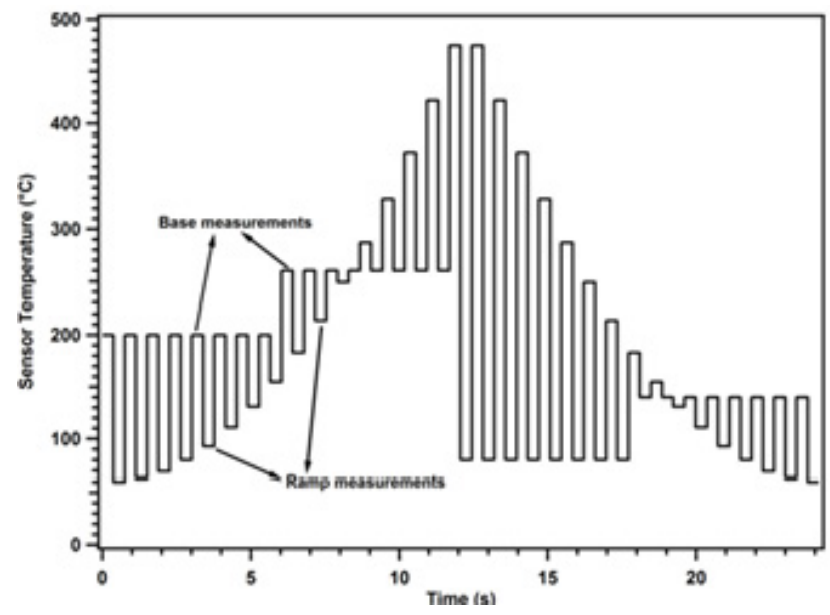

Figure 2. Temperature program with 32 ramp temperatures and four baseline temperatures used in this study for probing the $\mathrm{SnO}_{2}$ sensor response to various analytes. handling manifold, and includes a controlled exhaust for continuously maintaining a 1 standard liter per minute $(\mathrm{slm})$ flow rate.

\section{B. Data Analysis Methods}

Conductance measurements on the $\mathrm{SnO}_{2}$ sensor elements for the complete cycle of 32 ramp temperatures were concatenated to form a multi-dimensional sensor response that was used in all analyses. To detect the onset of a foreground odor and register it to the most recent baseline/background response, we employ the following algorithm:

1. Compute the correlation coefficient across sensor responses measured at the 32 different temperatures (1024 correlations) using ' $w$ ' consecutive measurements $(\mathrm{t}, \ldots, \mathrm{t}-\mathrm{w}+1)$ where $\mathrm{t}$ denotes the current measurement cycle.

2. If the computed standard deviation of the sensor response correlations between the 32 temperatures during the current measurement cycle $(\mathrm{t})$ and the previous cycle $(\mathrm{t}-1)$ are less than 'thresh' and the standard deviation for the cycle $(\mathrm{t}-3)$ is greater than thresh, then record the average response of the sensor array at cycle $t-3, \ldots, t-3-n$ as the most recent baseline.

3. Measure the response of the sensor device with respect to the most recent baseline. Then the corrected current response is $\mathrm{R}_{\text {new }}=\left(\mathrm{R}-\mathrm{R}_{0}\right)$, where $\mathrm{R}$ is the current measured response, and $\mathrm{R}_{0}$ is the most recently identified baseline response.

The following parameters were used to generate results described in this manuscript: $w=5$; thresh $=0.1 ; n=2$. Since both stimulus onsets and offsets were detectable using this approach, we enforced a fixed temporal window between two chemical events to limit baseline registration to chemical onsets.

For visualization purposes, linear discriminant analysis was used to reduce the dimensionality of the mulitvariate sensor responses. 


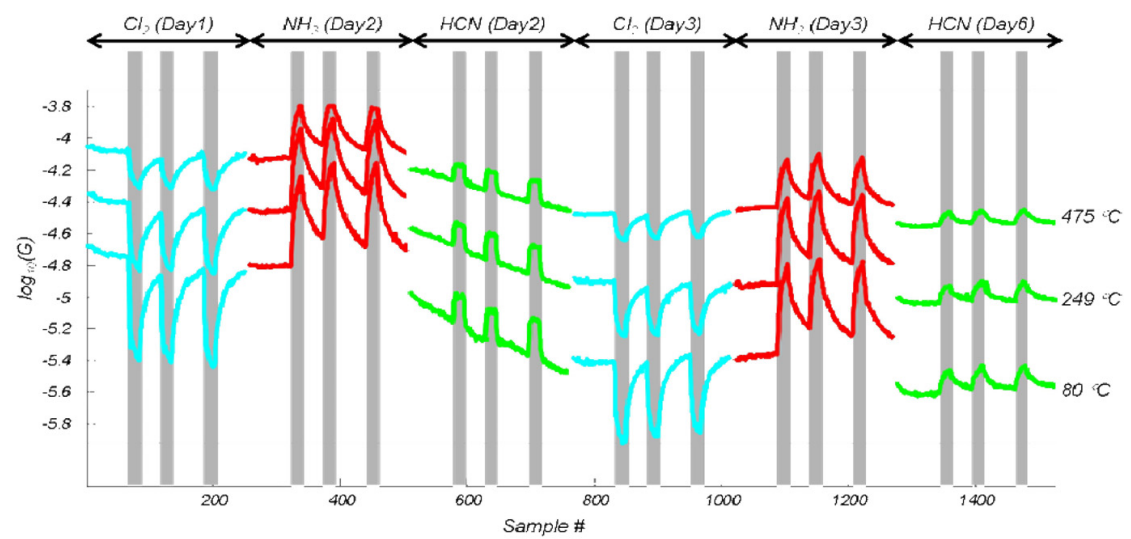

Figure 3. Selected illustrative responses of the $\mathrm{SnO}_{2}$ sensor (plotted as isotherms) at three different temperatures shown over a six-day data-collection period.

\section{RESUlTS AND DisCUSSION}

Fig. 3 illustrates the conductance measurements (plotted as log conductance) obtained from one of the $\mathrm{SnO}_{2}$ microsensor films (shown in Fig. 1b, 1c) at three different ramp temperatures. Note that introduction of reducing gases, $\mathrm{NH}_{3}$ and $\mathrm{HCN}$, both produce conductance increases, while the oxidizing species $\mathrm{Cl}_{2}$ produces a decrease in $\mathrm{SnO}_{2}$ conductance, for each of the six exposures included in the sampling set. It can be observed that although data taken over the six-day period reveal repeatable relative conductance changes to the three target analytes, a significant shift occurs in the response baseline. Hence, for successful detection/recognition of the targets, it is necessary to filter the baseline response from the analyte-induced conductance changes.

In order to track critical signal changes and (then) remove contributions to baseline shifts arising from age-induced changes or background compositional changes in an on-line manner, we examined a moving-window event detection approach based on correlation coefficients. Fig. 4 shows an example of correlation coefficients (along vertical axis) against experimental time (horizontal axis) which ranges over three exposures of the target analyte $(\mathrm{HCN})$; high-correlation

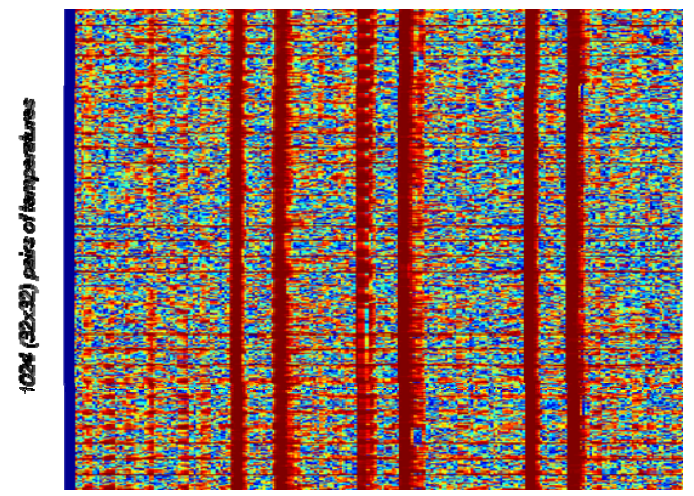

Figure 4. Event detection with temperature programmed sensors. Correlation coefficient between the 32 ramp-temperature measurements (1024 combinations; vertical axis) are shown over time (along the horizontal axis). The six high correlation bands correspond to alternating $\mathrm{HCN}$ onsets and offsets. bands were noticed at both the analyte ons ets and offsets. Similar onset/offset banding was also observed for both $\mathrm{NH}_{3}$ and $\mathrm{Cl}_{2}$. The mean correlation was high during "chemical events" such as target onset/offset, and the standard deviation remained low during the TIC exposure period. Thus a correlation mean increase, or a corresponding decrease in the standard deviation, can be used to register the time mark on the baseline response. The contribution of the problematic baseline/background in the signal can be effectively removed by re-computing sensor responses with respect to that most recently tagged baseline. While this method readily registers events that feature sharp chemical onsets/offsets (rapid concentration introduction and withdrawal), we also validated the robustness of this approach on more gradual analyte introductions [17].

A demonstration of the baseline-correction approach on raw conductance data is shown in Fig. 5. The red circles indicate the baseline just before the identified chemical events (i.e. - HCN introductions). By re-computing the sensor response with respect to the most recently registered baseline, the offset contribution is effectively removed (Fig. 5b).

Fig. 6 shows the multivariate sensor responses over a period of days after LDA dimensionality reduction for all targets. Only the training data for the three analytes were used to determine the projection vectors. Clearly the three analytes form well-defined, distinguishable clusters indicating that sufficient analytical information is available from the temperature-programmed sensor responses to both identify and distinguish the three targets in a 30\% RH background.

Next, we examined the ability of the approach to remove the contribution of an untrained background/interference. For this purpose, we introduced the targets in backgrounds that included vapors of either Windex [14], diesel or Clorox [14]. Here again, we adopted the same baseline correction approach to register and remove the sensor response to the background chemical. Fig. 7 shows an example of the response of the sensor during training (30\% RH background) and testing phases (here for the Windex-containing [14] background, with $30 \% \mathrm{RH})$ after dimensionality reduction. It is worth noting that the projection vectors were obtained using the response of the targets in 30\% RH background (training samples) alone. Clearly, the three targets form well-defined, separable clusters 

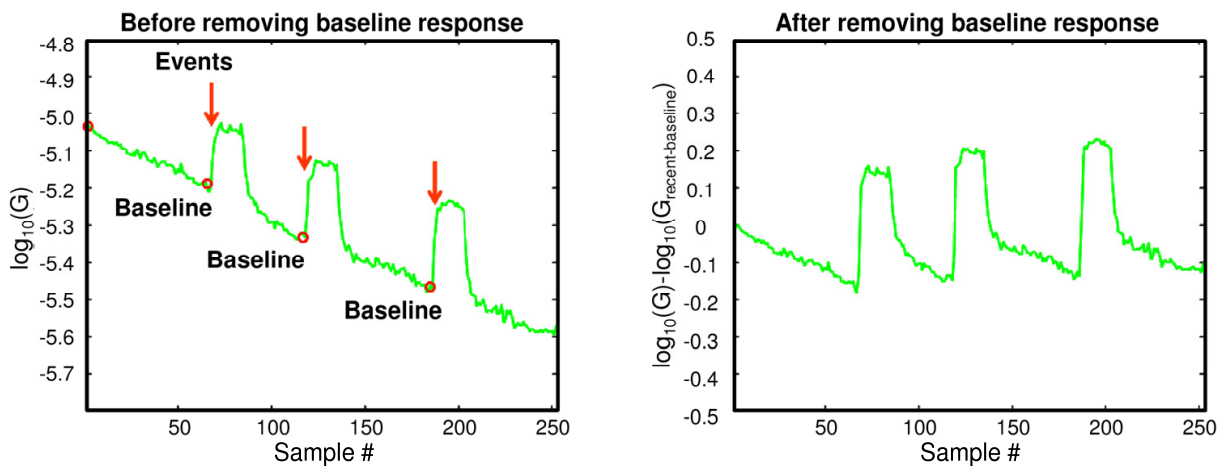

Figure 5. Demonstration of the online baseline correction approach. (a) Raw data with red circles denoting the HCN onsets detected by our approach. (b) The corrected baseline response.

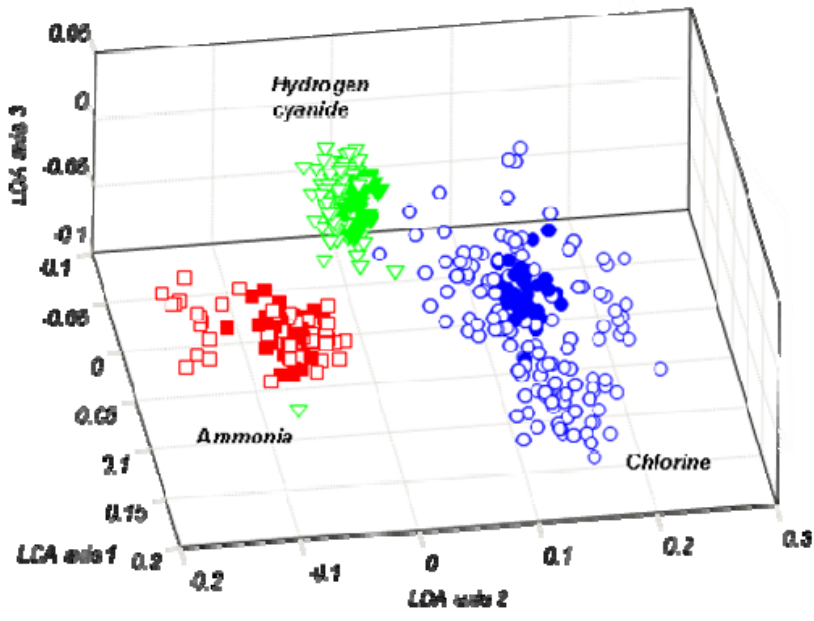

Figure 6. Recognition over time. LDA plot showing the dimensionality reduced response of a $\mathrm{SnO}_{2}$ sensor to the three targets (filled symbols, training samples: circles $-\mathrm{Cl}_{2}$, square $-\mathrm{NH}_{3}$ and triangle $-\mathrm{HCN}$ ) and up to 5 days after training data collection phase (open symbols, test phase measurements).

after the background contribution is removed from the sensor response.

\section{CONCLUSION}

We have demonstrated an event-detection and baselinecorrection methodology for separating foreground signals representative of a target species from background signals that can be strongly influenced by interfering compounds. In case studies involving temperature-modulated, chemiresistive microsensors, the approach succeeded in identifying IDLH levels of $\mathrm{NH}_{3}, \mathrm{HCN}$ and $\mathrm{Cl}_{2}$ even in air-based backgrounds that contained high concentrations of fuel and cleaning compounds that had not been present in prior training trials. During the course of development, we also showed that the baseline corrections can be effective in dealing with aging effects of the sensors. Ongoing work seeks to extend the capabilities of our methods to dynamic changes in backgrounds with unknown interferences, and also demonstrate approaches for reducing baseline differences in the electrical characteristics of microdevices fabricated under equivalent manufacturing procedures.

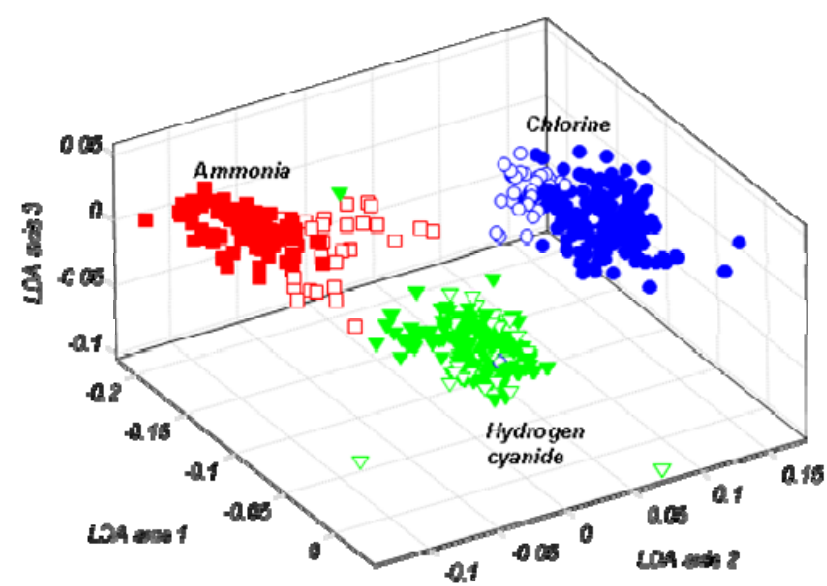

Figure 7. Recognition in an untrained Windex-containing [14] background. LDA plot showing the dimensionality reduced response of a $\mathrm{SnO}_{2}$ sensor to the three targets in $30 \% \mathrm{RH}$ background (filled symbols, training samples: circles $-\mathrm{Cl}_{2}$, square $-\mathrm{NH}_{3}$ and triangle $-\mathrm{HCN}$ ) and in Windex background (open symbols, test phase measurements).

\section{ACKNOWLEDGMENT}

We acknowledge partial financial support of this project by the U.S. Department of Homeland Security, Science and Technology Directorate. BR was supported by a NIH(NIBIB)NIST Joint Postdoctoral Associateship Award administered through the National Research Council. We also thank Mike Carrier and Chip Montgomery for their valuable contributions to this project.

\section{REFERENCES}

[1] N. Yamazoe, T. Fuchigami, M. Kishitawa and T. Seiyama, "Interactions of the tin oxide surface with $\mathrm{O}_{2}, \mathrm{H}_{2} \mathrm{O}$ and $\mathrm{H}_{2}$," Surf. Sci., vol. 86, pp.335-344, 1979.

[2] S. Semancik and R. E.Cavicchi, "Kinetically-controlled chemical sensing using micromachined structures," Acc. Chem. Res., vol. 31, pp. 279-287, 1998

[3] T. A. Kunt, T. J. McAvoy, R. E. Cavicchi and S. Semancik, "Optimization of temperature programmed sensing for gas identification using micro-hotplate sensors," Sensors and Actuators B, vol. 53 , pp. 24-43, 1998. 
[4] N. Barsan, U. Weimar, "Conduction model of metal oxide gas sensors," Journal of Electroceramics, vol. 7, pp. 143-167, 2001.

[5] J. Suehle, R. E. Cavicchi, M. Gaitan and S. Semancik, "Tin oxide gas sensor using micro-hotplate by CMOS technology and in-situ processing," IEEE-Electron Device Lett., vol. 14, pp. 118-120, 1993.

[6] S. Semancik, R. E. Cavicchi, M. C. Wheeler, J. E. Tiffany, G. E. Poirier, R. M. Walton, J. S. Suehle, B. Panchapakesan, and D. L. Devoe, "Microhotplate platforms for chemical sensor research," Sensors and Actuators, vol. B 77, pp. 579-591, 2001.

[7] M. Y. Afridi, J. S. Suehle, M. E. Zaghloul, D. W. Berning, A. R. Hefner, R. E. Cavicchi, S. Semancik, C. J. Taylor, and C. B. Montgomery, "A monolithic CMOS microhotplate-based gas sensor system," IEEE Sensors Journal, vol. 2, 644-655, 2002.

[8] R. Gutierrez-Osuna and A. Gutierrez-Galvez, "Habituation in the KIII olfactory model with chemical sensor arrays," IEEE Transactions on Neural Networks, vol. 14, pp. 1565-1568, 2003.

[9] A. A. Tomchenko, G. P. Harmer, and B. T. Marquis, "Detection of chemical warfare agents using nanostructured metal-oxide sensors," Sensors and Actuators B, vol. 108, pp. 41-55, 2005.

[10] R. Gutierrez-Osuna and N. U. Powar, "Odor mixtures and chemosensory adaptation in gas sensor arrays," International Journal on Artificial Intelligence Tools, vol. 12, pp. 1-16, 2003.

[11] B. Raman and R. Gutierrez-Osuna, "Mixture segmentation and background suppression in chemosensory arrays with a model of olfactory bulb-cortex interaction," in International Joint Conference on Neural Networks, Montreal, Canada, 2005, pp. 131-136.

[12] B. Raman, D. Meier, J. Evju, and S. Semancik, "Designing and optimizing microsensor arrays for recognizing chemical hazards in complex environments," Sensors and Actuators B, vol. 137, pp. 617$629,2009$.

[13] D. C. Meier, J. K. Evju, Z. Boger, B. Raman, K. D. Benkstein, C. J. Martinez, C. B. Montgomery, and S. Semancik, "The potential for and challenges of detecting chemical hazards with temperatureprogrammed microsensors," Sensors and Actuators B, vol. 121, pp. 282-294, 2006.

[14] Mention of these and any other commercial products is strictly for provision of proper experimental definition, and does not constitute an endorsement by the National Institute of Standards and Technology.

[15] S. Semancik, "Temperature-dependent materials research with micromachined array platforms," in Combinatorial Materials Synthesis, X. D. Xiang and I. Takeuchi, Eds. New York: Marcel Dekker, Inc., 2003, pp. 263-295.

[16] R. E. Cavicchi, S. Semancik, F. DiMeo and C. J. Taylor, C.J., "Use of microhotplates in the controlled growth and characterization of metal oxides for chemical sensing," J. of Electroceramics, vol. 9, pp.155-164, 2003.

[17] B. Raman, R. Shenoy, D. C. Meier, K. D. Benkstein and S. Semancik, "Recognizing chemicals in untrained backgrounds," Sensors and Actuators B, manuscript in preparation. 